\title{
Schizont transcriptome variation among clinical isolates and laboratory-adapted clones of the malaria parasite Plasmodium falciparum
}

Sarah J. Tarr ${ }^{1 *}$, Ofelia Díaz-Ingelmo ${ }^{1}$, Lindsay B. Stewart ${ }^{1}$, Suzanne E. Hocking ${ }^{1}$, Lee Murray ${ }^{1}$, Craig W. Duffy ${ }^{1}$, Thomas D. Otto ${ }^{2,3}$, Lia Chappell ${ }^{3}$, Julian C. Rayner ${ }^{3}$, Gordon A. Awandare ${ }^{4}$ and David J. Conway ${ }^{1 *}$ (D)

\begin{abstract}
Background: Malaria parasites are genetically polymorphic and phenotypically plastic. In studying transcriptome variation among parasites from different infections, it is challenging to overcome potentially confounding technical and biological variation between samples. We investigate variation in the major human parasite Plasmodium falciparum, generating RNA-seq data on multiple independent replicate sample preparations of merozoite-containing intra-erythrocytic schizonts from a panel of clinical isolates and from long-term laboratory-adapted clones, with a goal of robustly identifying differentially expressed genes.

Results: Analysis of biological sample replicates shows that increased numbers improve the true discovery rate of differentially expressed genes, and that six independent replicates of each parasite line allowed identification of most differences that could be detected with larger numbers. For highly expressed genes, focusing on the top quartile at schizont stages, there was more power to detect differences. Comparing cultured clinical isolates and laboratoryadapted clones, genes more highly expressed in the laboratory-adapted clones include those encoding an AP2 transcription factor (PF3D7_0420300), a ubiquitin-binding protein and two putative methyl transferases. In contrast, higher expression in clinical isolates was seen for the merozoite surface protein gene $d b / \mathrm{msp2}$, proposed to be a marker of schizonts forming merozoites committed to sexual differentiation. Variable expression was extremely strongly, but not exclusively, associated with genes known to be targeted by Heterochromatin Protein 1. Clinical isolates show variable expression of several known merozoite invasion ligands, as well as other genes for which new RT-qPCR assays validate the quantitation and allow characterisation in samples with more limited material. Expression levels of these genes vary among schizont preparations of different clinical isolates in the first ex vivo cycle in patient erythrocytes, but mean levels are similar to those in continuously cultured clinical isolates.
\end{abstract}

Conclusions: Analysis of multiple biological sample replicates greatly improves identification of genes variably expressed between different cultured parasite lines. Clinical isolates recently established in culture show differences from long-term adapted clones in transcript levels of particular genes, and are suitable for analyses requiring biological replicates to understand parasite phenotypes and variable expression likely to be relevant in nature.

Keywords: Eukaryotic microbial genomics, Biological replicates, Clinical samples, Culture adaptation, RNA-seq, Transcriptomic methods, Cell invasion, Immunity

\footnotetext{
* Correspondence: sarah.tarr@lshtm.ac.uk; david.conway@lshtm.ac.uk

${ }^{1}$ Pathogen Molecular Biology Department, London School of Hygiene and

Tropical Medicine, London, UK

Full list of author information is available at the end of the article
}

(c) The Author(s). 2018 Open Access This article is distributed under the terms of the Creative Commons Attribution 4.0 International License (http://creativecommons.org/licenses/by/4.0/), which permits unrestricted use, distribution, and reproduction in any medium, provided you give appropriate credit to the original author(s) and the source, provide a link to the Creative Commons license, and indicate if changes were made. The Creative Commons Public Domain Dedication waiver (http://creativecommons.org/publicdomain/zero/1.0/) applies to the data made available in this article, unless otherwise stated. 


\section{Introduction}

Variation in gene expression is key to survival and reproduction of microbes, affecting diverse phenotypes such as sexual differentiation [1], adaptation to different growth conditions [2] and evasion of host immunity [3]. Malaria pathogenesis occurs as parasites undergo cycles of invasion and asexual replication inside erythrocytes. Towards the end of each cycle, schizonts develop which contain multiple merozoites that need to burst from the host cell and invade new erythrocytes. The parasite transcriptional program of the major human malaria parasite Plasmodium falciparum is highly synchronised throughout the asexual replication cycle in erythrocytes [4], but some variation exists between parasite clones $[5,6]$. Analysis of naturally occurring polymorphism in P. falciparum has shown that selection maintains multiple alleles of many merozoite-stage genes [7, 8], some of which encode targets of naturally acquired immunity [9-11]. Genes expressed at this stage can also exhibit apparently pronounced variation in transcription among parasites [7, 12-14], but most analyses have been conducted on sample preparations that do not incorporate many biological replicates, so that the extent of variation among different parasite lines is unclear.

Accurate quantitation of differential gene expression between organisms or groups of organisms in whole-transcriptome analyses requires biological replicate sampling $[15,16]$. The importance of minimising sampling error in order to detect real differential gene expression is widely recognised [17], and some bioinformatic tools can guide the determination of replicate numbers appropriate to experimental designs $[18,19]$. In transcriptomic studies of malaria parasites, achieving large numbers of replicate sample preparations is difficult, particularly from clinical isolates [5], and most detailed understanding of transcription has been derived from a small number of parasite lines that have been grown in culture for many years. Although long-term culture-adapted parasites are phenotypically and transcriptionally diverse $[6,12,20]$, it is not clear to what extent they reflect the diversity of parasites causing clinical malaria. Examination of genome sequences of culture-adapted parasites has identified premature stop codon mutants affecting some transcription factor genes and cell signalling protein genes [21, 22], as well as particular gene deletions and amplifications not seen in parasites sampled directly from patients [23-25].

Studies of parasite transcripts in clinical isolates face a challenge of interpreting the mixture of developmental stages that may be present in a blood sample, with parasites at different stages of the asexual cycle as well as parasites that have committed to sexual development [26-28]. Some of the unknown variation in parasite stage distribution may be accounted for using deconvolution methods in analysis [28, 29], and some random variation may be partly overcome statistically if it is possible to analyse large numbers of infections [30, 31]. However, P. falciparum parasites at the intra-erythrocytic schizont stage, containing merozoites ready to be released to invade other erythrocytes, are normally sequestered in organ capillaries and not present to be analysed in blood samples. This stage of the parasite may be studied by ex vivo development in the first cycle of following isolation from patients, and analysis of matured schizont stages has shown variable expression of particular genes [7, 13, 14], although the precision of such analyses are limited without biological replicate measurements.

Here we present gene expression profiles of schizont-stage malaria parasites from recently culture-established clinical isolates, and laboratory-adapted clones previously cultured for many years. We conduct RNA-seq analysis with multiple replicates of each parasite line, and show that high numbers of biological replicates improves the true-positive discovery rate for identifying differentially expressed genes. This identifies schizont-stage genes that have differing transcript levels between the long-term laboratory-adapted clones and cultured clinical isolates, as well as those variably expressed among the clinical isolates. The results confirm variable transcription in particular genes encoding ligands involved in erythrocyte invasion, and variation in expression of genes that have been less characterised was quantitatively validated by targeted RT-qPCR assays, allowing analysis to be extended to additional samples from which material is more limited. The expression levels of these genes in a panel of clinical isolates cultivated to schizont stage in the first ex vivo cycle were similar on average to those in continuously cultured clinical isolates. This encourages use of recently culture-established parasite lines for studies requiring biological replication.

\section{Results \\ Biological replicate sampling improves detection of differentially expressed genes}

RNA-seq paired-end short-read sequences from all samples were mapped to the $P$. falciparum strain 3D7 reference genome sequence, and regions of genes with high levels of allelic polymorphism were masked from analysis in order to minimize mapping bias among the different parasite lines (Additional file 1). Genes previously described as deleted in some parasites were not excluded, as most individual deletions are very rare [32] and exclusion would result in unnecessary loss of data for genes unaffected in the isolates sampled. After excluding the subtelomeric large gene families var, rifin and stevor for which short read sequence mapping is not generally locus-specific, a total of 5134 gene loci were analysed. 
We assessed the impact of numbers of biological replicates on detection of genes with differing transcript levels between parasite lines, by first analysing RNA-seq data for the 3D7 and D10 laboratory clones, focusing on schizonts purified by density gradient centrifugation from ten different culture preparations of each clone. Analysing data from all ten replicates identified 123 genes with adjusted $P$ values $<0.01$ and $\log _{2}$ differences of $>2$ in relative transcript levels between the two clones (equivalent to at least 4-fold differences, Additional file 2). We assessed what proportions of these 123 genes were captured as being differentially expressed in 100 random samples of two, four, six and eight replicates within each group. The true-positive proportion of differentially expressed genes detected (using the same statistical criteria of absolute $\log _{2}$ fold difference $>2$ and adjusted $P$ value $<0.01)$ increased with the number of replicates within each group, median true-positive proportions being $0.28,0.52,0.67$ and 0.79 , for two, four, six and eight replicates respectively (Fig. 1). These true-positives accounted for the majority of genes indicated as differentially expressed, out of median totals of 42, 81, 108

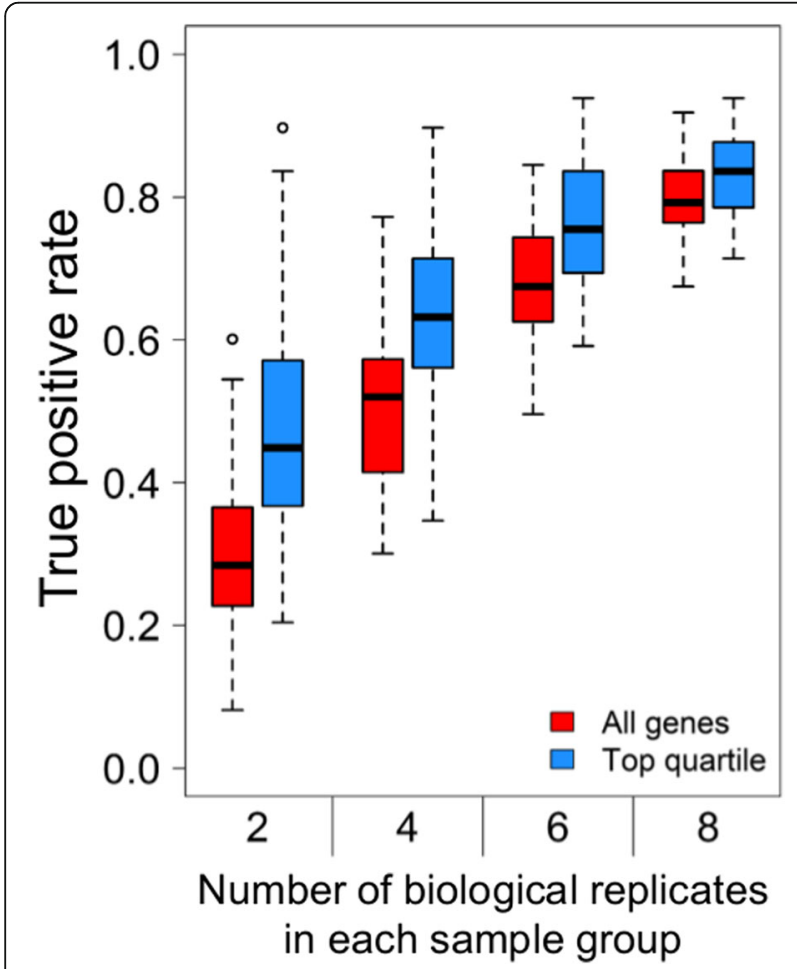

Fig. 1 Increasing numbers of sample replicates improves identification of schizont-stage genes varying in expression between different $P$. falciparum lines. Assessment of the proportion of genes captured as being differentially expressed between two different parasite clones (3D7 and D10), by taking 100 random samples of two, four, six and eight replicates of each (out of ten initially analysed replicates that identified 123 genes with $\log _{2}$ differences of $>2$ in relative transcript levels between the two clones) and 111 genes, for two, four, six and eight replicates respectively. The median false-positive rates were very low in all cases (among the genes that did not show differences with 10 replicates, the proportions showing apparent differences with two, four, six and eight replicates were $0.001,0.004,0.005$ and 0.004 respectively).

We also examined the sensitivity to detect differential expression when focusing specifically on genes that are highly expressed in schizont-stage samples, as these include genes most likely to be functionally important for the merozoite invasive stage. The FPKM values for each sample in each of the comparisons were averaged and the genes were ranked by their maximum expression level. The top quartile of most highly expressed genes were analysed for differences between clone 3D7 and D10, in all ten replicates and subsamples of lower replicate numbers, with 100 randomisations of each. In comparison with all ten replicates, there were median true-positive rates of $0.45,0.63,0.78$ and 0.86 for comparisons respectively containing two, four, six and eight replicates, higher proportions than for the analysis of all genes (Fig. 1). Based on these data, it is evident that increasing biological replicate numbers for parasite RNA-seq improves discovery, and under our experimental conditions the use of six independent biological replicates detects the majority of genes shown to be differentially expressed using ten replicates.

\section{Comparison of gene expression in cultured clinical and laboratory-adapted isolates}

In addition to the ten biological replicate preparations from each of clones 3D7 and D10 analysed above, another nine biological replicate preparations of schizonts from clone 3D7 were analysed, as well as seven biological replicates for each of two other laboratory-adapted clones (HB3 and Dd2), and five or six replicates of each of six cultured clinical isolates from Ghana (isolates 217, 278, 280, 286, 293 and 296: Additional file 3). Despite being enriched for mature schizont stages, multiple biological replicates cannot each have exactly the same distribution of parasite developmental stages, so to assess stage variability the RNA-seq read depth for each gene (FPKMs) for each replicate sample were correlated with FPKM values in reference RNA-seq data for seven cultured time points over a $\sim 48 \mathrm{~h} P$. falciparum cycle [33]. This identified only a small number of replicate samples that did not have a maximum Spearman's correlation with parasites at schizont stages (either 40 or $48 \mathrm{~h}$ post-invasion), and these were excluded from further analyses (Additional file 3). Although there is variation evident among the remaining replicates that were analysed, within each isolate the FPKMs among replicates correlated highly, with average Spearman's $\rho>0.95$ for pairwise correlations across all genes (Fig. 2). 


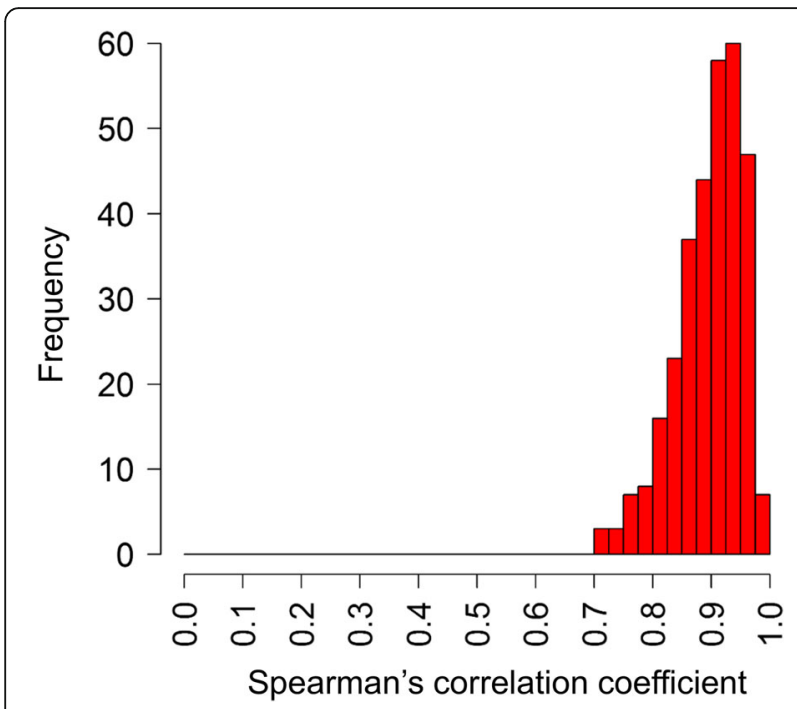

Fig. 2 Distribution of correlations of FPKM expression values among biological sample replicate transcriptomes for each isolate or clone. The final numbers of replicates, and median Spearman's correlation coefficients among replicates were: $3 \mathrm{D} 7 n=19, \rho=0.89 ; \mathrm{D} 10 n=10$, $\rho=0.93 ; \mathrm{Dd} 2 n=6, \rho=0.95 ; \mathrm{HB} 3 n=7, \rho=0.89 ; 271 n=3, \rho=0.94$; $278 n=5, \rho=0.91 ; 280 n=6, \rho=0.93 ; 286 n=6, \rho=0.97 ; 293 n=6$, $\rho=0.93 ; 296 n=3, \rho=0.90$

In an overall comparison of the schizont preparations from four long-term laboratory-adapted clones and six cultured clinical isolates, 132 genes $(2.6 \%$ of those analysed) appeared to have different transcript levels between the two groups, each having $\log _{2}$ fold difference > 2 and adjusted $P$ value $<0.01$ (Additional file 4). Among the genes within the top quartile of expression values overall, 18 genes $(1.4 \%)$ had such a difference between the groups, a lower proportion than among the rest of the genes (Odds Ratio 0.52, Fisher's exact $P=0.006$ ). Examining the 18 genes with differing transcript levels among the top quartile of expressed genes, several were seen to contain a deletion in one of the parasite lines or to be members of multigene families. For example, PF3D7_1371600 (ebl-1) and PF3D7_0935700 (near the end of chromosome 9) are deleted in the HB3 strain and D10 strain respectively (Fig. 3), as previously documented [34, 35]. After excluding these genes and others for which one or more of the samples were presumed to contain genetic deletions, and excluding members of sub-telomeric multi-gene families, ten highly expressed single-locus genes showed differences between laboratory and clinical isolates (Table 1).

There was considerable variation in the measurements for individual genes among the different biological replicate preparations of each parasite clone or isolate, with five to ten-fold ranges of levels being not unusual (Fig. 3), illustrating the importance of sampling and analysing multiple biological replicates. To check for potential effects of fine differences in schizont maturity among samples, expression levels of the ten most differentiated genes were compared between samples having overall highest correlations with either the 40-h or the 48-h time points of a reference transcriptome dataset [33]. This showed that the gene expression distributions were not due to differences in estimated maturity, and that differences between clinical isolates and laboratory clones remained when only analysing replicates having peak correlation with the 40-h time point (Additional file 5).

Eight of the ten highly expressed genes showing a difference between the groups had higher transcript levels in the long-term laboratory-adapted clones than in the cultured clinical isolates (Table 1). These include an AP2 transcription factor gene PF3D7_0420300, as well as two predicted methyltransferase genes (PF3D7_0422900 and PF3D7_0522300), and gene PF3D7_0104300 encoding ubiquitin-binding protein 1 which is involved in protein turnover [36] (Fig. 3). The genes that had higher transcript levels in clinical isolates were PF3D7_1036300 which encodes the merozoite surface protein MSPDBL2 (Fig. 3), and PF3D7_1302100 which encodes the gamete antigen $27 / 25$. MSPDBL2 has previously been shown to be only expressed in a minority of schizonts in culture, with clone HB3 having more positive schizonts than other laboratory-adapted clones [7], consistent with the higher transcript level in HB3 seen here and previously. Recent experimental analysis has shown $m s p d b l 2$ transcript levels to be markedly increased in parasite cultures when function of heterochromatin protein 1 (HP1) is suppressed by gametocyte development protein 1 (GDV1) leading to gametocytogenesis [37, 38]. Although gamete antigen 27/ 25 had higher transcript levels in clinical isolates and was previously shown to be expressed in early gametocytes $[38,39]$ another gene PF3D7_1327300 that is transcribed in gametocytes [40] showed lower expression in the clinical isolates (Table 1).

We investigated whether there was an overall association between the genes differentially expressed and targets of HP1 regulation [37, 41]. Of the HP1-regulated genes previously identified [37], 118 genes are among those analysed here (var, rifin and stevor gene families were excluded). Of the 132 genes differentially expressed between the two groups, 24 (18.2\%) were targets of HP1 regulation, a higher proportion than among the rest of the genes (Odds Ratio 11.3 , Fisher's exact $P=1.3 \times 10^{-5}$ ).

\section{Differential expression of schizont-stage genes among cultured clinical isolates}

Overall, 214 genes $(4.2 \%)$ showed a $\log _{2}$ fold difference $>2$ for at least one of the 15 pairwise comparisons among cultured clinical isolates. Of those genes in the top quartile of expression levels, 39 genes (3.0\%) showed a difference for at least one pairwise comparison, a lower proportion than 


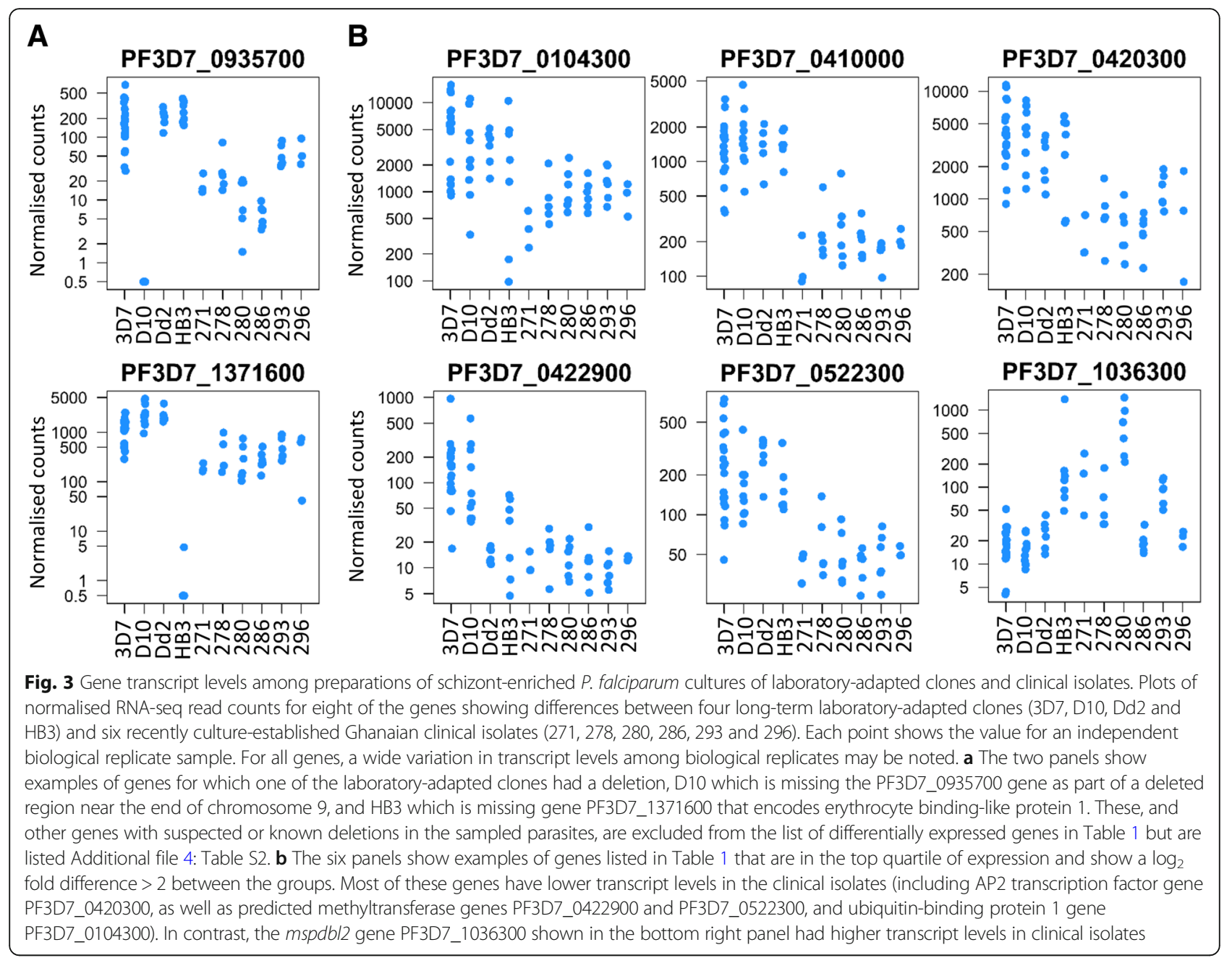

Table $1 P$. falciparum genes in the top quartile of expression showing a difference in schizont stage transcript levels between longterm laboratory-adapted clones and recent clinical isolates in culture

\begin{tabular}{|c|c|c|c|}
\hline Gene ID & Product annotation & $\log _{2}$ fold difference & Wald test \\
\hline \multicolumn{4}{|c|}{ Higher transcription in long-term laboratory-adapted clones } \\
\hline PF3D7_0422900 & methyltransferase, putative & -2.50 & -6.48 \\
\hline PF3D7_0501300 & skeleton-binding protein 1 & -2.50 & -4.98 \\
\hline PF3D7_0410000 & erythrocyte vesicle protein 1 & -2.38 & -10.62 \\
\hline PF3D7_0104300 & ubiquitin carboxyl-terminal hydrolase 1, putative & -2.18 & -6.13 \\
\hline PF3D7_0522300 & $18 \mathrm{~S}$ rRNA (guanine-N(7))-methyltransferase, putative & -2.15 & -8.67 \\
\hline PF3D7_0420300 & AP2 domain transcription factor, putative & -2.02 & -7.19 \\
\hline PF3D7_1030200 & claudin-like apicomplexan microneme protein, putative & -2.00 & -9.21 \\
\hline PF3D7_1327300 & conserved Plasmodium protein, unknown function & -2.00 & -4.97 \\
\hline \multicolumn{4}{|c|}{ Higher transcription in clinical isolates } \\
\hline PF3D7_1036300 & duffy binding-like merozoite surface protein 2 & 3.02 & 5.32 \\
\hline PF3D7_1302100 & gamete antigen $27 / 25$ & 2.02 & 3.99 \\
\hline
\end{tabular}


among the less expressed genes (Odds Ratio 0.66, Fisher's Exact $P=0.02$ ) (Additional file 6). Some of the variably expressed genes encode merozoite proteins previously characterised as transcriptionally variable among ex vivo clinical isolates [14], including dblmsp 2 and $m s p 6$, as well as erythrocyte binding antigen genes eba-175, eba-181, and $e b a-140$ (which is also deleted in one of the laboratory adapted clones) (Additional file 7). Notably, among the 214 genes variably expressed among cultured clinical isolates, 38 (17.8\%) have been shown to be targets of HP1 regulation, compared with only $1.6 \%$ of the non-variably expressed genes (Odds Ratio 12.9, Fisher's Exact $P<2.2 \times 10^{-16}$ ). This association was even more extreme among genes in the top quartile of overall expression values, 16 (41\%) of the 39 variably expressed genes being targets of HP1 regulation compared with only $0.6 \%$ of the non-variably expressed genes (Odds Ratio 118.6, Fisher's exact $P<2.2 \times 10^{-16}$ ).

To validate the data obtained through RNA-seq, a subset of genes was selected for quantitation by reverse-transcription quantitative PCR (RT-qPCR). We chose differentially expressed genes encoding proteins containing predicted transmembrane domains or signal peptides, thereby likely to enter the parasite secretory pathway, excluding genes previously characterised as variably expressed or members of multi-gene families. Eight genes were selected for assay (Additional file 8), two of which encode known antigens (merozoite-associated tryptophan-rich antigen [42] and liver-stage antigen-3 [43]). These gene transcripts were quantified by RT-qPCR in 49 RNA preparations from the laboratory clones and clinical isolates that had been analysed by RNA-seq. Transcript levels were normalised to those of a housekeeping gene (serine-tRNA ligase PF3D7_0717700) and correlated with similarly normalised FPKM expression values for the RNA-seq data. Each gene showed strong positive and highly significant correlation between RNA-seq and RT-qPCR-derived expression measures, all except one having correlation coefficients $>0.8$ (Fig. 4).

\section{Variable expression among isolates with schizonts sampled in the first ex vivo cycle}

We next determined the expression levels in additional clinical samples from Ghana matured during the first cycle ex vivo until a large proportion of parasites were schizonts. For nine of these isolates there was sufficient RNA yield for RNA-seq to be performed, out of which seven gave adequate data for transcriptome analysis. With remaining material from two of these as well as another six isolates there was sufficient to analyse the selected eight gene panel by RT-qPCR. Characterisation of the ex vivo samples showed considerable variation in relative expression levels of each gene, but the mean levels across the isolates were similar to those determined in the continuously-cultured parasites that had extensive sample replication (Fig. 5). Without sample replication of the first cycle ex vivo clinical samples,

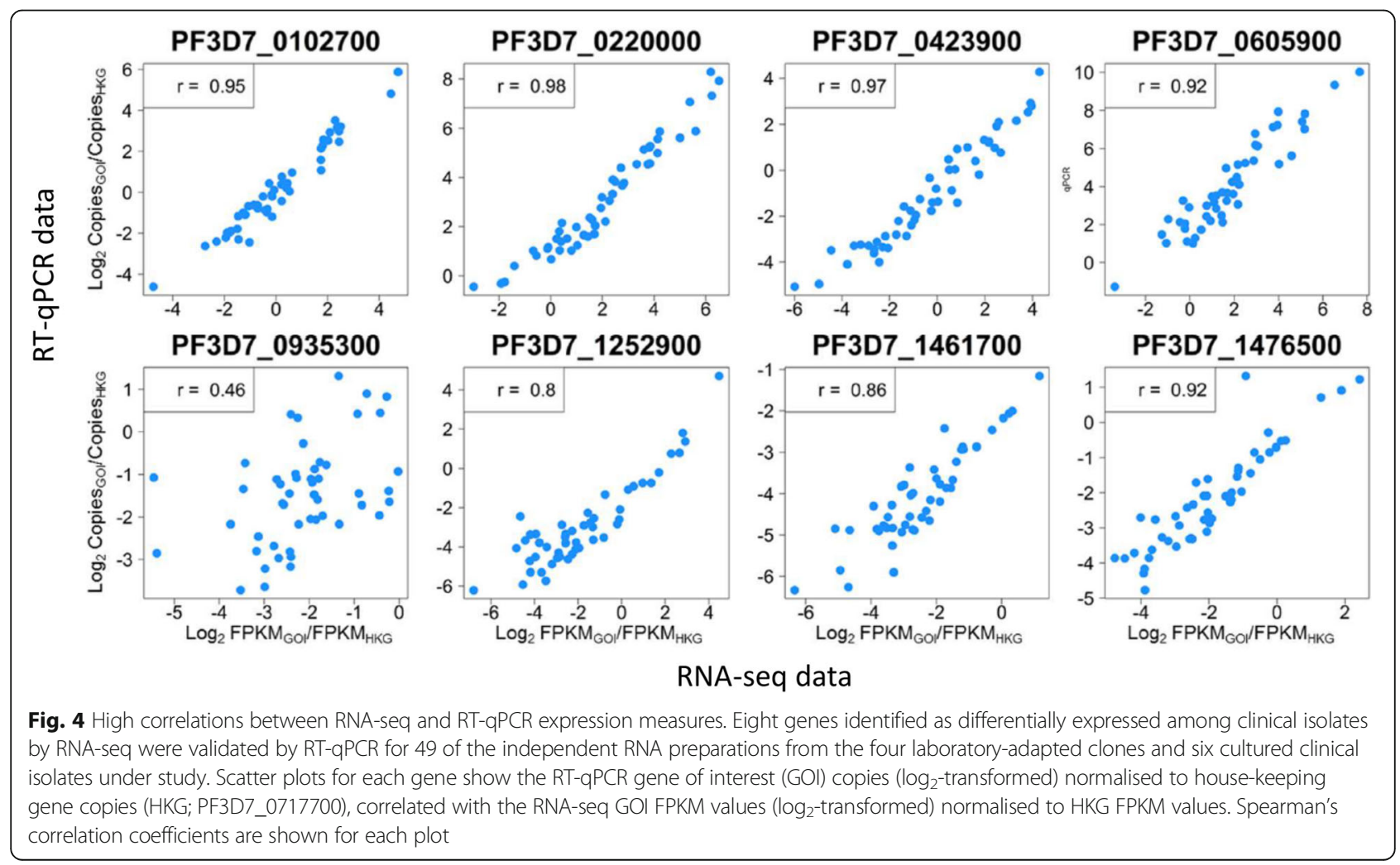


routine technical variation and random sampling effects are likely to exaggerate the apparent variation in expression levels. However, the observation that for each gene the normalised expression values have similar means to those for cultured clinical lines indicates there are no systematic differences in expression between the first ex vivo cycle and under continuous culture conditions. These results support the use of cultured clinical isolates as a means of studying parasite gene expression reflecting original populations, while enabling the necessary experimental replication for many analyses.

\section{Discussion}

Transcriptomic analyses perform optimally with increased levels of sample replication to minimise the impact of stochastic or technical variation in individual samples [17, 44]. This is particularly important for studies focusing on a defined developmental stage, as errors may be caused by subtle differences between samples. However, the nature of clinical samples of malaria parasites is such that replication is technically very difficult. In order to assess the degree of replication useful to identify differentially expressed genes, we first generated RNA-seq profiles from multiple replicates of laboratory-adapted clones, and used the data to calculate the true- and false-discovery rates obtained using fewer replicates. Consistent with studies on other cultured eukaryotes [17], we show that for malaria parasites increasing the sample replication improves the true-positive discovery rate in identifying variably expressed genes, and under the experimental conditions here six independent replicates were useful to balance accuracy and experimental feasibility. Where these numbers of replicates are not obtainable, it is recommended to focus on genes that are highly expressed in order to achieve as much accuracy as possible per gene. Conversely, where a very high sensitivity is needed it may be worth studying even more biological replicates for each parasite line and condition being investigated.

It has been proposed that spontaneous transcriptional variation within parasite populations is a strategy that ensures fitness of parasites facing a range of potential and changing selective pressures [6], and it is likely that the rates and mechanisms of regulation differ among most genes. In comparison between long-term laboratory-adapted clones and cultured clinical isolates, we found that among the genes most differentially expressed were an AP2-transcription factor [45] and two methyltransferases, the functional roles of

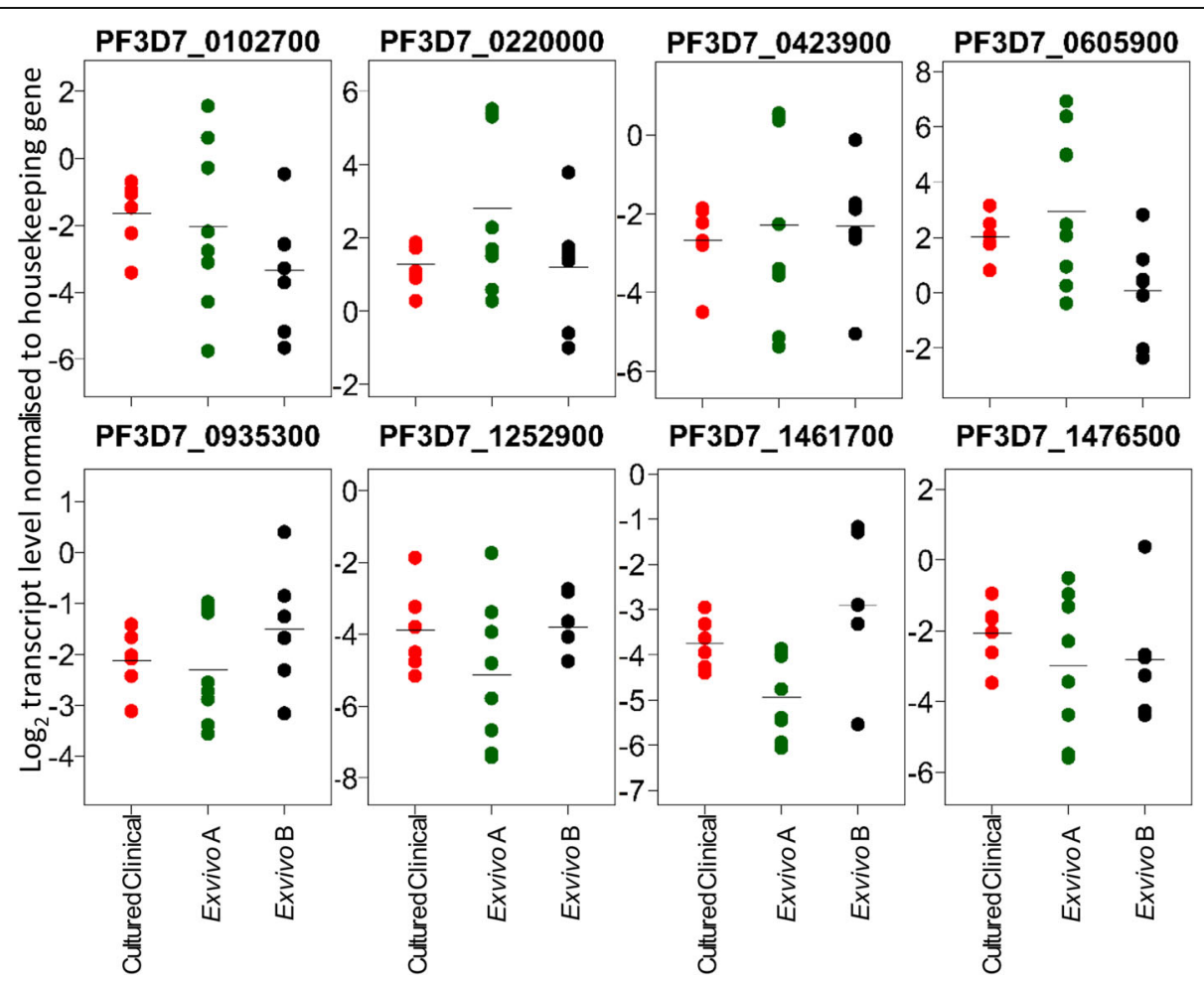

Fig. 5 Transcript levels of variably expressed genes for first cycle ex vivo clinical samples compared with continuously cultured isolates. For each gene, the $\log _{2}$ normalised expression value is plotted as a ratio of the value for a housekeeping gene (PF3D7_0717700). For the cultured clinical isolates (red, $N=6$ ), each point represents the mean expression value for each gene calculated across multiple replicates of each isolate. For the ex vivo clinical isolates, each point represents values for single samples without replicates. The panel of ex vivo A samples (green $N=8)$ were analysed by RT-qPCR, while the panel of ex vivo B samples (black, $N=7$ ) were analysed by RNA-seq 
which remain to be determined although genome-wide mutagenesis studies indicate they are important for $P$. falciparum asexual erythrocytic stage replication [46]. It is possible that use of different oxygen concentrations in the culture of long-term adapted clones and recent clinical isolates here might affect transcription of some genes, particularly in earlier trophic stages of the intra-erythrocytic cycle [47], and although we did not consider oxygen metabolism in analysis of schizont stages it could be examined in future. Most of the differentially expressed genes noted here had lower transcription in the clinical isolates, and we note that HP1 is responsible for extensive gene silencing in malaria parasites [38], and there was an over representation of HP1-regulated genes among those differentially expressed between laboratory-adapted clones and cultured clinical isolates. It would be interesting to undertake studies directly on HP1 and other chromatin modifications in recent clinical isolates, as experimental studies on chromatin have so far focused on long-term laboratory-adapted clones. Interestingly, among the top quartile of expressed genes, the one most markedly highly expressed in clinical isolates was mspdbl2 that encodes a merozoite surface protein expressed within only a minority of schizonts [7] which may be a marker of parasites committed to sexual development [37]. Further studies of clinical isolates are needed to understand the process of parasite commitment to sexual differentiation and transmission, as indicated by other transcriptome comparisons $[5,30]$.

We identified many genes differentially expressed among the clinical isolates. Targeted RT-qPCR assays of schizont-stage ex vivo cultured clinical isolates have previously shown some of the genes to be differentially expressed $[7,13,14]$. Focusing on genes for which variable expression has not been previously studied, we selected a panel of eight highly differentially expressed genes that encode secreted proteins. Of these, the merozoite-associated tryptophan-rich antigen and liver stage antigen 3 have been previously identified as merozoite proteins [42, 43], and another gene (PF3D7_0423900) is adjacent to loci encoding the cysteine-rich protective antigen (CyRPA) and reticulocyte binding homologue 5 (Rh5) which are targets of antibodies that inhibit merozoite invasion. We extensively validated the RNA-seq data by RT-qPCR for these genes, and extended quantitation to additional ex vivo clinical isolate samples. Despite the lack of sample replicates for the latter, results showed that average expression levels for each gene were similar to those for the cultured samples that had biological replicates.

Together, these data highlight new candidates for investigation as potential determinants of alternative developmental pathways or targets of immunity. Characterisation of gene transcript levels is only one level of analysis, as proteomic characterisation [48] and analysis of phosphorylation and other protein modifications $[49,50]$ may also identify functional determinants, and large-scale analysis of parasite protein variation has not yet been attempted on clinical isolates. A more complete description of transcriptome variation may also be derived in future by analysis of individual infected cells, which has not been undertaken on parasites from clinical isolates, as initial single-cell sequencing studies of laboratory model malaria parasites have only recently begun to probe the expression pathways of different life-cycle stages [51-53]. Scaling up transcriptomic analyses to clinical studies is challenging, and biological replicate preparations of parasite samples may only be feasible in a limited number of cases. In an epidemiological context, reduction of error also requires large sample sizes and tight definition of clinical phenotypes, and more statistical analysis and modelling of covariates may be appropriate, ideally including data on host transcriptome variation within the same samples [54].

\section{Conclusions}

The transcriptomes of Plasmodium falciparum erythrocytic schizont preparations of long-term laboratory-adapted clones and recently established clinical isolates were analysed, using large numbers of biological replicate samples to minimise the impact of inter-replicate variation on observed patterns of differential expression. Testing subsamples of up to ten replicates of each cultured line shows that six replicate preparations gives power to detect most differences. Particular genes show differential expression in laboratory-adapted clones compared to cultured clinical isolates, or among different clinical isolates, including those predicted to affect differentiation and encoding targets of immunity. In schizonts grown in the first cycle ex vivo, expression levels of a panel of these genes vary among isolates, but mean levels are similar to those in continuously cultured clinical isolates suitable for experimental analyses requiring biological replicates to understand relevant parasite phenotypes.

\section{Methods}

\section{P. falciparum isolates sampled from clinical malaria cases}

Blood samples were collected from clinical malaria cases attending Ghana government health facilities between 2012 and 2013, in Kintampo (Brong-Ahafo Region of central Ghana), and Navrongo (Kassena-Nankana East Municipality, in the Upper East Region of northern Ghana). Patients were eligible to participate in the study if they had uncomplicated clinical malaria, were aged 2-14 years, tested positive for $P$. falciparum malaria by lateral flow rapid diagnostic test and slide microscopy, and had not taken antimalarial drugs during the $72 \mathrm{~h}$ preceding sample collection. Venous blood samples (up to $5 \mathrm{ml}$ ) were collected into heparinized Vacutainer tubes (BD Biosciences). Blood samples were centrifuged, plasma separated 
and leukocyte buffy coat cell layer removed, and erythrocytes were cryopreserved in glycerolyte and stored frozen at $-80^{\circ} \mathrm{C}$ or in liquid nitrogen until shipment on dry ice to the London School of Hygiene and Tropical Medicine for subsequent culture.

\section{Parasite culture and enrichment for schizont stages}

Parasites from ex vivo clinical samples were matured within the original patient erythrocytes at $2-5 \%$ haematoctit for up to $48 \mathrm{~h}$ in RPMI 1640 medium containing $2 \%$ human $A B$ serum (GE Healthcare) and $0.3 \%$ Albumax II (Thermo Fisher Scientific) under an atmosphere of $5 \% \mathrm{O}_{2}, 5 \% \mathrm{CO}_{2}$, and $90 \% \mathrm{~N}_{2}$ at $37^{\circ} \mathrm{C}$, until most parasites were at the schizont stage of parasite development. Laboratory-adapted clones and continuously cultured clinical isolates were grown in erythrocytes at $2-5 \%$ haematocrit in RPMI 1640 medium containing 0.5\% Albumax II, at $37^{\circ} \mathrm{C}$. The biological replicate schizont preparations of clinical isolates for RNA-seq analysis were made by sampling at multiple time points between 182 and 250 days after first initiation into continuous culture. Laboratory-adapted clones which have been in culture for many years (3D7, HB3, Dd2, and D10) were maintained under atmospheric air with $5 \% \mathrm{CO}_{2}$, and cultured clinical isolates were maintained in $5 \% \mathrm{O}_{2}, 5 \% \mathrm{CO}_{2}, 90 \% \mathrm{~N}_{2}$. Schizonts were purified using magnetic-activated cell sorting (MACS) for all of the lines, except for the D10 and HB3 clones and ten of the 3D7 replicates which were purified using Percoll density gradient centrifugation.

For MACS purification of cultured clinical isolates, parasites were prepared from $100 \mathrm{ml}$ cultures with at least $0.7 \%$ of erythrocytes containing schizonts. Parasites were isolated by magnetic purification using magnetic LD Separation columns (Miltenyi Biotech). One column was used per $25 \mathrm{ml}$ culture, and first washed twice in $3 \mathrm{ml}$ of culture medium at room temperature. Parasite culture erythrocytes were pelleted by centrifugation at $500 \mathrm{~g}$ for 5 $\mathrm{min}$, then re-suspended in $3 \mathrm{ml}$ culture medium per $1 \mathrm{ml}$ of packed cell volume. The re-suspended material was bound to the MACS column, which was then washed three times with $3 \mathrm{ml}$ culture medium, and schizonts were eluted twice by removing the magnet from the column and forcing $2 \mathrm{ml}$ culture medium through the column into a $15 \mathrm{ml}$ collection tube. Finally, the schizonts were pelleted by centrifugation at $500 \mathrm{~g}$ for $5 \mathrm{~min}$ and the pellet volume was estimated, with $0.5 \mu \mathrm{l}$ used for Giemsa-stained microscopical examination to assess staging, $1 \mu$ ladded back to $250 \mu \mathrm{l}$ culture at $0.8 \%$ hematocrit to follow the progression. Remaining parasites were re-suspended in $1.5 \mathrm{ml}$ of culture medium with $10 \mu \mathrm{M}$ E64 in a 12 well plate, and parasites were incubated for $5.5 \mathrm{~h}$ in $5 \% \mathrm{CO}_{2}$ at $37^{\circ} \mathrm{C}$, before centrifugation in a $1.5 \mathrm{ml}$ tube and processing for RNA extraction.
For discontinuous density centrifugation purification, parasites were maintained as $25 \mathrm{ml}$ cultures at $2.5 \%$ hematocrit. Cultures were used when $>1 \%$ erythrocytes contained parasites with multiple nuclei. Schizonts were purified using $70 \%$ Percoll (GE Healthcare) $/ 2.93 \%$ sorbitol/PBS overlayed with 35\% Percoll/1.47\% sorbitol/PBS, which was overlayed with parasitized erythrocytes, allowing the schizonts to be separated by centrifugation at $2500 \mathrm{~g}$ for $10 \mathrm{~min}$ at $24{ }^{\circ} \mathrm{C}$, with a light brake at the end of centrifugation. Purified schizonts were washed once in complete medium and the pellet volume was estimated, following which the pellet was resuspended with six times the pellet volume of $50 \%$ haematocrit erythrocytes, a slide was prepared for microscopic examination, and the cells were then incubated in $6 \mathrm{ml}$ of complete culture medium. Of this, $1 \mathrm{ml}$ was used as a control untreated sample to track parasite egress, and $10 \mu \mathrm{M}$ final concentration of E64 was added to the remaining $5 \mathrm{ml}$, and both were cultured at $37^{\circ} \mathrm{C}$ in a $5 \%$ $\mathrm{CO}_{2}$ static incubator for $5.5 \mathrm{~h}$. Schizonts from the E64-treated culture were overlaid on 70\% Percoll/2.93\% sorbitol/PBS and separated by centrifugation at $2500 \mathrm{~g}$ for $10 \mathrm{~min}$ at $24^{\circ} \mathrm{C}$, with a light brake at the end of centrifugation. The schizont layer was washed once in complete culture medium and final cell pellets of approximately $10-20 \mu \mathrm{l}$ were used for RNA extraction.

\section{RNA extraction}

Erythrocytes containing the prepared schizonts were resuspended in $500 \mu \mathrm{IRIzol}^{\circ}$ reagent (Thermo Fisher Scientific) and stored at $-80^{\circ} \mathrm{C}$ until RNA extraction using RNeasy mini columns (Qiagen). RNA pellets were dissolved in $100 \mu \mathrm{l}$ RNase-free $\mathrm{H}_{2} \mathrm{O}$, and a second RNA clean-up and on-column DNase treatment was carried out with RNA eluted in 30-50 $\mu \mathrm{l}$ RNase-free $\mathrm{H}_{2} \mathrm{O}$, and concentration quantified by Qubit High Sensitivity RNA Assay (Thermo Fisher Scientific). Samples containing at least 500 ng RNA were considered for RNA-seq processing after the RNA integrity was checked on an Agilent Bioanalyzer using RNA 6000 Nano reagents and chips (Agilent Genomics).

\section{RNA-seq library preparation and sequencing}

RNA-seq libraries of each of the replicate preparations of parasites were prepared with TruSeq Stranded mRNA Library Prep Kits (Illumina) using $500 \mathrm{ng}-1 \mu \mathrm{g}$ RNA following the Illumina TruSeq Stranded mRNA protocol. Libraries were validated on an Agilent Bioanalyzer using DNA 1000 reagents and chips (Agilent Genomics) to quantify library sizes and confirm the absence of primer dimers. Libraries were quantified using a KAPA Universal Library Quantification kit (Roche Diagnostics Limited) on a 7500 Fast Real-Time PCR System (Thermo Fisher Scientific) and library concentrations 
were adjusted for library size. $12-15$ pM pooled libraries were sequenced on a MiSeq System (Illumina) using a MiSeq Reagent Kit v3 (Illumina) with $2 \times 75$ cycles.

RNA-seq libraries of ex vivo schizont-enriched $P$. falciparum isolates (not cultured beyond the first cycle) were prepared using a modified protocol. PolyA+ RNA (mRNA) was selected using magnetic oligo-d(T) beads, and mRNA was reverse transcribed using Superscript III $^{\oplus}$ (Thermo Fisher Scientific), primed using oligo-d(T) primers, with dUTP included during second-strand cDNA synthesis. The resulting double stranded cDNA was fragmented using a Covaris AFA sonicator. Sheared double stranded cDNA was dA-tailed, end repaired, and "PCR-free" barcoded sequencing adaptors (Bioo Scientific) [55] were ligated. Libraries were cleaned twice, using solid phase reversible immobilisation beads, and eluted in EB buffer (Qiagen). Second strand cDNA was removed using uracil-specific excision reagent enzyme mix (NEB) prior to library generation, and libraries were assayed by quantitative PCR prior to sequencing on a HiSeq system (Illumina).

\section{Data analysis}

Raw Illumina sequence reads were aligned to the $P$. falciparum 3D7 v3 genome using HISAT2 [56] and converted to 'BAM' format using SAMtools [57]. Reads with MAPQ scores < 60 were removed. Reads were counted using the "summarizeOverlaps" feature of the GenomicAlignments package [58] in $\mathrm{R}$, against a previously published $P$. falciparum genome annotation file that had been masked for regions of polymorphism (outlined in Additional file 1, which explains the coded annotation in Additional files 9 and 10). Fragments Per Kilobase of transcript per Million mapped reads (FPKMs) for the data here and those of previously published data on a developmental time course of the clone 3D7 [33] were calculated using the 'fpkm' function of DESeq2 [59] in R. FPKMs for all genes in each of our parasite preparations were correlated using a Spearman's Rank correlation with FPKMs for each of the seven time points $(0,8,16,24,32,40$ and $48 \mathrm{~h}$ post-invasion; Additional file 2: Table S1). Replicates with peak Spearman correlation values of $\rho>0.7$ at the latest time points $(40$ or $48 \mathrm{~h}$ ) were included for further analysis. Differential expression analysis was conducted using DESeq2 in $\mathrm{R}$.

To test that the protocol for E64 treatment to prevent schizont egress from erythrocytes had no major effect on the transcriptomes, a comparison of four paired replicates of schizont preparations of cultured $P$. falciparum clone 3D7 was performed, which showed that only a single gene had $\log _{2}$ fold difference $>2$ (Additional file 11), a difference considered as potentially random and marginal compared to differences anticipated between different parasite lines. The E64 treatment as described above enabled efficient preparation of mature schizonts, so this was used for preparations of schizonts throughout the study.

\section{RT-qPCR assays}

150-500 ng total RNA from each preparation of parasite schizonts was reverse transcribed using Superscript $\mathrm{II}^{\circ}$ (Thermo Fisher Scientific) with $250 \mathrm{ng}$ random hexamer oligonucleotide primers per $20 \mu \mathrm{l}$ reaction. Quantitative PCR (qPCR) was carried out using $\mathrm{SYBR}^{\bullet}$ Select Master Mix (Thermo Fisher Scientific) with $500 \mathrm{nM}$ forward and reverse primers, in a Prism 7500 Fast qPCR machine. For each gene, threshold-cycle values were quantified against a serially diluted genomic DNA (Dd2 strain) standard curve, run on the same plate. Cycling parameters were: $50^{\circ} \mathrm{C}$ for $2 \mathrm{~min}, 95^{\circ} \mathrm{C}$ for $2 \mathrm{~min}$ followed by 40 cycles of $95^{\circ} \mathrm{C}$ for $15 \mathrm{~s}$ and $60{ }^{\circ} \mathrm{C}$ for $1 \mathrm{~min}$. All wells were run as $10 \mu \mathrm{l}$ volumes in technical duplicate. The qPCR copy numbers were normalised against copies of a house-keeping gene, PF3D7_0717700 [60]. PCR primer pair sequences are as follows: PF3D7_0102700 5'-CAACCAGACAAACAACAAGAAA-3' and 5'-TCCAT TCTGATGCTTTCCAC-3', PF3D7_0220000 5'-GTAAAT GGTGAATTAGCTAGTGAAG-3' and 5'CCTTTATATC TTCGGCTTCTTCT-3', PF3D7_0423900 5'-GAGAAGAA GCCAAAGTAAATGAAC-3' and 5'-GAATGTGTCAAG TGCATCATAA-3', PF3D7_0605900 5'CGCAATAACAAGAAGTCACAAA-3' and 5'-AAGATTGTAGGAGGGTG TTAAT-3', PF3D7_0935300 5'-GGGCTGCTGTTATACC 'TTG-3' and 5'-AGAATGAGGAGGTTCAGTTTG-3', PF3 D7_1252900 5'-CCTTAGTAGAACTTCAAAGTGACA-3' and 5'-TGTAACCAACTACGAAATTCCC-3', PF3D7_14 61700 5'-TGCTGACTTTCAAGAGATAAGG-3' and 5'-T TTCATTTGTTCATTTTGTACAACC-3', PF3D7_147650 0 5'-CTTCGATTCACAAATGCAGAAA-3' and 5'-CGTA TTCCACACCTTGTTCTAT-3', PF3D7_0717700 5'-AAGTAGCAGGTCATCGTGGTT-3' and 5'-GTTCGGCACAT TCTTCCATAA-3'.

\section{Additional files} Additional file 1: Masking of $P$. falciparum genome annotation file to
exclude polymorphic regions of genes. (PDF $546 \mathrm{~kb}$ )

Additional file 2: Table S1. List of genes with different detected transcript levels between 3D7 and D10 strains by absolute log2 fold > 2 (at least 4 fold difference). Note that this list contains some genes that are deleted in either line. There are also two highly polymorphic genes that were not masked in the initial annotation file, asterisked below $\left(^{*}\right)$ and excluded from subsequent analyses. (XLSX $18 \mathrm{~kb}$ )

Additional file 3: Figure S1. Multiple biological replicate preparations of P. falciparum schizont stage transcriptomes in correlation with reference stage-specific transcriptome data. Multiple parasite preparations were made from four long-term laboratory adapted clones (between seven and ten replicates of each) and six Ghanaian clinical isolates (five or six replicates of each) for RNA-seq analysis. Each parasite culture was enriched for schizontstage parasites, with egress blocked using E64 treatment. Plots show the correlations of FPKM values across all genes in comparisons with previous data from seven time-points across the $P$. falciparum asexual erythrocytic 
cycle [33], with peak correlations indicating the predominant parasite stage in each replicate. Red lines plot data for samples with peak correlation at either 40 or $48 \mathrm{~h}$ post-invasion, and grey lines plot replicate samples that did not maximally correlate with either of these time points, which were therefore excluded from further analysis. (TIFF $92 \mathrm{~kb}$ )

Additional file 4: Table S2. Genes showing significant differences in transcript levels (log 2 fold $>2$, at least 4 fold difference on average) in comparison of six cultured clinical isolates and four long-term laboratory adapted clones, including all biological replicate samples. For genes among the top quartile of expression values genome-wide (top 18 genes in the table), members of multigene families and genes in which strain-specific deletions may be responsible for differences are annotated with asterisks (*). (XLSX $20 \mathrm{~kb}$ )

Additional file 5: Figure S2. Normalised read counts for the ten most highly differentiated genes between cultured clinical isolates and laboratoryadapted clones. Individual sample replicates are plotted according to the time of overall peak transcriptome correlation with reference time course data (either 40 or $48 \mathrm{~h}$ ). Replicates from clinical isolates are in red (those having peak correlation with $40 \mathrm{~h}$ are plotted), and replicates from laboratory isolates are in blue. (PDF $312 \mathrm{~kb}$ )

Additional file 6: Table S3. $\log _{2}$ fold differences in transcript levels of genes differentially expressed among pairwise comparisons of six cultured clinical isolates with multiple schizont preparations of each, among genes within the top quartile of expression overall. (XLSX $24 \mathrm{~kb})$

Additional file 7: Figure S3. Differential expression of merozoite invasion-related genes among schizonts from different parasite cultures. Distributions of read counts (normalised to library size) for eight genes, for replicated laboratory-adapted and clinical isolate samples, showing data from each replicate culture preparation of each strain. (PDF $324 \mathrm{~kb}$ )

Additional file 8: Figure S3. Gene expression levels for eight genes newly detected as differentially expressed among clinical isolates (Table 1). Distributions of read counts (normalised to library size) for eight genes, showing data from each replicate culture preparation of each strain. (PDF $84 \mathrm{~kb}$ )

Additional file 9: Sequence annotation file Pf3D7.May2015.NoSplice.LSHTM.gtf (see Additional file 1 for explanatory details). (GTF $2322 \mathrm{~kb}$ )

Additional file 10: Sequence annotation file GTF VarRifStev filtered out.gtf (see Additional file 1 for explanatory details). (GTF $2102 \mathrm{~kb}$ )

Additional file 11: RNA-seq comparison of gene expression in paired E64treated and untreated P. falciparum 3D7 schizont preparations. (PDF 365 kb)

\section{Acknowledgements}

We are grateful to malaria patients who contributed blood samples for analysis of parasite transcription, and to colleagues who supported the clinical sample collection and initial laboratory processing. We are also grateful to Mandy Sanders for support in sequencing some of the samples, and to Alistair Miles and Antoine Claessens for help towards identification of genomic coordinates of highly polymorphic sequences within genes.

\section{Funding}

Funding for the study was granted by the European Research Council (AdG-2011294428, https://erc.europa.eu/) to DJC, the Leverhulme Trust-Royal Society (AA110050, https://royalsociety.org/) to GAA and DJC, the Biotechnology and Biological Sciences Research Council (BBSRC, London Interdisciplinary Doctoral Programme https://bbsrc.ukri.org/) to LM, SEH and DJC, and the Wellcome Trust (098051, https://wellcome.ac.uk) to JCR. The funders had no role in study design, data collection and analysis, decision to publish, or preparation of the manuscript.

\section{Availability of data and materials}

The datasets supporting the conclusions of this article, including the read count matrices, are available in the Gene Expression Omnibus (https://www.ncbi.nlm.nih.gov/geo/), entry: GSE113718 for the P. falciparum RNA-seq data from the laboratory-adapted and cultured clinical isolates, and in the European Nucleotide Archive (https://www.ebi.ac.uk/ena), study: ERP103955 for the P. falciparum RNA-seq data from the first cycle ex vivo isolates.

\section{Authors' contributions}

SJT contributed to technical design, performed parasite culture, RNA-seq data analysis and drafted the manuscript. OD-I contributed to technical design, performed parasite culture and RNA-seq data generation. LBS contributed to technical design, performed parasite culture and RNA-seq data generation. SEH, LM, and CWD assisted with RNA-seq bioinformatics data analysis. TDO advised and gave input on RNA-seq bioinformatics data analysis and interpretation. LC performed RNA-seq data generation. JCR contributed to technical design, RNAseq data generation and manuscript revision. GAA contributed to technical design, managed sample collection and contributed to manuscript revision. DJC designed and supervised the study, contributed to technical design and RNA-seq data analysis and interpretation, and managed manuscript drafting and revision. All authors read and approved the final manuscript.

\section{Ethics approval}

This study of clinical parasite transcriptomes was approved by the Ethics committees of the Ghana Health Service, the Noguchi Memorial Institute for Medical Research, University of Ghana, the Kintampo Health Research Centre, the Navrongo Health Research Centre and the London School of Hygiene and Tropical Medicine. Written informed consent was obtained from parents or legal guardians of participating children with malaria, and additional assent was received from the children.

\section{Consent for publication}

All authors declare consent for publication.

\section{Competing interests}

The authors declare that they have no competing interests.

\section{Publisher's Note}

Springer Nature remains neutral with regard to jurisdictional claims in published maps and institutional affiliations.

\section{Author details}

${ }^{1}$ Pathogen Molecular Biology Department, London School of Hygiene and Tropical Medicine, London, UK. ${ }^{2}$ Institute of Infection, Immunity and Inflammation, University of Glasgow, Scotland, UK. ${ }^{3}$ Wellcome Sanger Institute, Hinxton, Cambridge, UK. ${ }^{4}$ West African Centre for Cell Biology of Infectious Pathogens, Department of Biochemistry, Cell and Molecular Biology, University of Ghana, Legon, Ghana.

Received: 21 August 2018 Accepted: 16 November 2018 Published online: 10 December 2018

\section{References}

1. Mata J, Wilbrey A, Bahler J. Transcriptional regulatory network for sexual differentiation in fission yeast. Genome Biol. 2007;8:R217.

2. Cases I, de Lorenzo V, Ouzounis CA. Transcription regulation and environmental adaptation in bacteria. Trends Microbiol. 2003;11:248-53.

3. Cestari I, Stuart K. Transcriptional regulation of telomeric expression sites and antigenic variation in trypanosomes. Curr Genomics. 2018;19:119-32.

4. Bozdech Z, Llinás M, Pulliam BL, Wong ED, Zhu J, de Risi JL. The transcriptome of the intraerythrocytic developmental cycle of $P$ falciparum. PLoS Biol. 2003;1:e5.

5. Mackinnon MJ, Li J, Mok S, Kortok MM, Marsh K, Preiser PR, Bozdech Z Comparative transcriptional and genomic analysis of Plasmodium falciparum field isolates. PLoS Pathog. 2009:5:e1000644.

6. Rovira-Graells N, Gupta AP, Planet E, Crowley VM, Mok S, Ribas de Pouplana $L$, Preiser PR, Bozdech Z, Cortes A. Transcriptional variation in the malaria parasite Plasmodium falciparum. Genome Res. 2012;22:925-38.

7. Amambua-Ngwa A, Tetteh KK, Manske M, Gomez-Escobar N, Stewart LB, Deerhake ME, Cheeseman IH, Newbold Cl, Holder AA, Knuepfer E, et al. Population genomic scan for candidate signatures of balancing selection to guide antigen characterization in malaria parasites. PLoS Genet. 2012;8:e1002992.

8. Ochola LI, Tetteh KK, Stewart LB, Riitho V, Marsh K, Conway DJ. Allele frequency-based and polymorphism-versus-divergence indices of balancing selection in a new filtered set of polymorphic genes in Plasmodium falciparum. Molr Biol Evol. 2010;27:2344-51. 
9. Osier FH, Mackinnon MJ, Crosnier C, Fegan G, Kamuyu G, Wanaguru M, Ogada E, McDade B, Rayner JC, Wright GJ, et al. New antigens for a multicomponent blood-stage malaria vaccine. Sci Trans Med. 2014;6:247ra102.

10. Richards JS, Arumugam TU, Reiling L, Healer J, Hodder AN, Fowkes FJ, Cross $\mathrm{N}$, Langer $C$, Takeo $S$, Uboldi AD, et al. Identification and prioritization of merozoite antigens as targets of protective human immunity to Plasmodium falciparum malaria for vaccine and biomarker development. J Immunol. 2013;191:795-809.

11. Tetteh KK, Osier FH, Salanti A, Kamuyu G, Drought L, Failly M, Martin C, Marsh K, Conway DJ. Analysis of antibodies to newly described Plasmodium falciparum merozoite antigens supports MSPDBL2 as a predicted target of naturally acquired immunity. Infect Immun. 2013;81:3835-42.

12. Stubbs J, Simpson KM, Triglia T, Plouffe D, Tonkin CJ, Duraisingh MT, Maier AG, Winzeler EA, Cowman AF. Molecular mechanism for switching of $P$. falciparum invasion pathways into human erythrocytes. Science. 2005;309:1384-7.

13. Gomez-Escobar N, Amambua-Ngwa A, Walther M, Okebe J, Ebonyi A, Conway DJ. Erythrocyte invasion and merozoite ligand gene expression in severe and mild Plasmodium falciparum malaria. J Infect Dis. 2010;201:444-52.

14. Bowyer PW, Stewart LB, Aspeling-Jones H, Mensah-Brown HE, Ahouidi AD, Amambua-Ngwa A, Awandare GA, Conway DJ. Variation in Plasmodium falciparum erythrocyte invasion phenotypes and merozoite ligand gene expression across different populations in areas of malaria endemicity. Infect Immun. 2015;83:2575-82.

15. Hansen KD, Wu Z, Irizarry RA, Leek JT. Sequencing technology does not eliminate biological variability. Nat Biotechnol. 2011;29:572-3.

16. Elowitz MB, Levine AJ, Siggia ED, Swain PS. Stochastic gene expression in a single cell. Science. 2002;297:1183-6.

17. Schurch NJ, Schofield P, Gierlinski M, Cole C, Sherstnev A, Singh V, Wrobel N, Gharbi K, Simpson GG, Owen-Hughes T, et al. How many biological replicates are needed in an RNA-seq experiment and which differential expression tool should you use? RNA. 2016;22:839-51.

18. Seo J, Gordish-Dressman H, Hoffman EP. An interactive power analysis tool for microarray hypothesis testing and generation. Bioinformatics. 2006;22:808-14.

19. Busby MA, Stewart C, Miller CA, Grzeda KR, Marth GT. Scotty: a web tool for designing RNA-Seq experiments to measure differential gene expression. Bioinformatics. 2013;29:656-7.

20. Llinas M, Bozdech Z, Wong ED, Adai AT, DeRisi JL. Comparative whole genome transcriptome analysis of three Plasmodium falciparum strains. Nucleic Acids Res. 2006;34:1166-73.

21. Claessens A, Affara M, Assefa SA, Kwiatkowski DP, Conway DJ. Culture adaptation of malaria parasites selects for convergent loss-of-function mutants. Sci Rep. 2017;7:41303.

22. Kafsack BF, Rovira-Graells N, Clark TG, Bancells C, Crowley VM, Campino SG, Williams AE, Drought LG, Kwiatkowski DP, Baker DA, et al. A transcriptional switch underlies commitment to sexual development in malaria parasites. Nature. 2014;507:248-52.

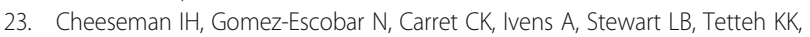
Conway DJ. Gene copy number variation throughout the Plasmodium falciparum genome. BMC Genomics. 2009;10:353.

24. Jeffares DC, Pain A, Berry A, Cox AV, Stalker J, Ingle CE, Thomas A, Quail MA, Siebenthall K, Uhlemann AC, et al. Genome variation and evolution of the malaria parasite Plasmodium falciparum. Nat Genet. 2007;39:120-5.

25. Nair S, Nkhoma S, Nosten F, Mayxay M, French N, Whitworth J, Anderson T. Genetic changes during laboratory propagation: copy number at the reticulocyte-binding protein 1 locus of Plasmodium falciparum. Mol Biochem Parasitol. 2010;172:145-8.

26. Daily JP, Scanfeld D, Pochet N, Le Roch K, Plouffe D, Kamal M, Sarr O, Mboup S, Ndir O, Wypij D, et al. Distinct physiological states of Plasmodium falciparum in malaria-infected patients. Nature. 2007;450:1091-5.

27. Pelle KG, Oh K, Buchholz K, Narasimhan V, Joice R, Milner DA, Brancucci NM, Ma S, Voss TS, Ketman K, et al. Transcriptional profiling defines dynamics of parasite tissue sequestration during malaria infection. Genome Medicine. 2015;7:19.

28. Lemieux JE, Gomez-Escobar N, Feller A, Carret C, Amambua-Ngwa A, Pinches R, Day F, Kyes SA, Conway DJ, Holmes CC, et al. Statistical estimation of cell-cycle progression and lineage commitment in Plasmodium falciparum reveals a homogeneous pattern of transcription in ex vivo culture. Proc Natl Acad Sci U S A. 2009;106:7559-64.

29. Joice R, Narasimhan V, Montgomery J, Sidhu AB, Oh K, Meyer E, Pierre-Louis W, Seydel K, Milner D, Williamson K, et al. Inferring developmental stage composition from gene expression in human malaria. PLoS Comp Biol. 2013;9:e1003392.

30. Rono MK, Nyonda MA, Simam JJ, Ngoi JM, Mok S, Kortok MM, Abdullah AS, Elfaki MM, Waitumbi JN, El-Hassan IM, et al. Adaptation of Plasmodium falciparum to its transmission environment. Nature Ecol Evol. 2017;1.

31. Mok S, Ashley EA, Ferreira PE, Zhu L, Lin Z, Yeo T, Chotivanich K, Imwong M, Pukrittayakamee S, Dhorda M, et al. Drug resistance. Population transcriptomics of human malaria parasites reveals the mechanism of artemisinin resistance. Science. 2015;347:431-5.

32. Cheeseman IH, Miller B, Tan JC, Tan A, Nair S, Nkhoma SC, De Donato M, Rodulfo H, Dondorp A, Branch OH, et al. Population structure shapes copy number variation in malaria parasites. Mol Biol Evol. 2016;33:603-20.

33. Otto TD, Wilinski D, Assefa S, Keane TM, Sarry LR, Bohme U, Lemieux J, Barrell B, Pain A, Berriman M, et al. New insights into the blood-stage transcriptome of Plasmodium falciparum using RNA-Seq. Mol Microbiol. 2010;76:12-24.

34. Peterson DS, Wellems TE. EBL-1, a putative erythrocyte binding protein of Plasmodium falciparum, maps within a favored linkage group in two genetic crosses. Mol Biochem Parasitol. 2000;105:105-13.

35. Nacer A, Roux E, Pomel S, Scheidig-Benatar C, Sakamoto H, Lafont F, Scherf A, Mattei D. Clag9 is not essential for PfEMP1 surface expression in noncytoadherent Plasmodium falciparum parasites with a chromosome 9 deletion. PLoS One. 2011;6:e29039.

36. Henriques $G$, Hallett RL, Beshir KB, Gadalla NB, Johnson RE, Burrow R, van Schalkwyk DA, Sawa P, Omar SA, Clark TG, et al. Directional selection at the pfmdr1, pfcrt, pfubp1, and pfap2mu loci of Plasmodium falciparum in Kenyan children treated with ACT. J Infect Dis. 2014;210:2001-8.

37. Filarsky M, Fraschka SA, Niederwieser I, Brancucci NMB, Carrington E, Carrio E, Moes S, Jenoe P, Bartfai R, Voss TS. GDV1 induces sexual commitment of malaria parasites by antagonizing HP1-dependent gene silencing. Science. 2018;359:1259-63.

38. Brancucci NMB, Bertschi NL, Zhu L, Niederwieser I, Chin WH, Wampfler R, Freymond C, Rottmann M, Felger I, Bozdech Z, et al. Heterochromatin protein 1 secures survival and transmission of malaria parasites. Cell Host Microbe. 2014;16:165-76

39. Carter R, Graves PM, Creasey A, Byrne K, Read D, Alano P, Fenton B. Plasmodium falciparum: an abundant stage-specific protein expressed during early gametocyte development. Exp Parasitol. 1989;69:140-9.

40. Lu XM, Batugedara G, Lee M, Prudhomme J, Bunnik EM, Le Roch KG. Nascent RNA sequencing reveals mechanisms of gene regulation in the human malaria parasite Plasmodium falciparum. Nucleic Acids Res. 2017; 45:7825-40.

41. Fraschka SA, Filarsky M, Hoo R, Niederwieser I, Yam XY, Brancucci NMB, Mohring F, Mushunje AT, Huang $X$, Christensen PR, et al. Comparative heterochromatin profiling reveals conserved and unique epigenome signatures linked to adaptation and development of malaria parasites. Cell Host Microbe. 2018;23:407-20.

42. Ntumngia FB, Bouyou-Akotet MK, Uhlemann AC, Mordmuller B, Kremsner PG, Kun JF. Characterisation of a tryptophan-rich Plasmodium falciparum antigen associated with merozoites. Mol Biochem Parasitol. 2004;137:349-53.

43. Morita M, Takashima E, Ito D, Miura K, Thongkukiatkul A, Diouf A, Fairhurst RM, Diakite M, Long CA, Torii M, et al. Immunoscreening of Plasmodium falciparum proteins expressed in a wheat germ cell-free system reveals a novel malaria vaccine candidate. Sci Rep. 2017;7:46086.

44. Robinson MD, Smyth GK. Moderated statistical tests for assessing differences in tag abundance. Bioinformatics. 2007;23:2881-7.

45. Balaji S, Babu MM, Iyer LM, Aravind L. Discovery of the principal specific transcription factors of Apicomplexa and their implication for the evolution of the AP2-integrase DNA binding domains. Nucleic Acids Res. 2005;33: 3994-4006.

46. Zhang M, Wang C, Otto TD, Oberstaller J, Liao X, Adapa SR, Udenze K, Bronner IF, Casandra D, Mayho M, et al. Uncovering the essential genes of the human malaria parasite Plasmodium falciparum by saturation mutagenesis. Science. 2018;360.

47. Duffy $S$, Avery VM. Routine in vitro culture of Plasmodium falciparum: experimental consequences? Trends Parasitol. 2018:34:564-75.

48. Kumar K, Srinivasan P, Nold MJ, Moch JK, Reiter K, Sturdevant D, Otto TD, Squires RB, Herrera R, Nagarajan V, et al. Profiling invasive Plasmodium falciparum merozoites using an integrated omics approach. Sci Rep. 2017;7:17146.

49. Doerig C, Rayner JC, Scherf A, Tobin AB. Post-translational protein modifications in malaria parasites. Nat Rev Microbiol. 2015;13:160-72. 
50. Jones ML, Collins MO, Goulding D, Choudhary JS, Rayner JC. Analysis of protein palmitoylation reveals a pervasive role in Plasmodium development and pathogenesis. Cell Host Microbe. 2012;12:246-58.

51. Poran A, Notzel C, Aly O, Mencia-Trinchant N, Harris CT, Guzman ML, Hassane DC, Elemento O, Kafsack BFC. Single-cell RNA sequencing reveals a signature of sexual commitment in malaria parasites. Nature. 2017;551:95-9.

52. Reid AJ, Talman AM, Bennett HM, Gomes AR, Sanders MJ, Illingworth CJR, Billker O, Berriman M, Lawniczak MK. Single-cell RNA-seq reveals hidden transcriptional variation in malaria parasites. eLife. 2018;7:e33105.

53. Walzer KA, Kubicki DM, Tang X, Chi JT. Single-cell analysis reveals distinct gene expression and heterogeneity in male and female Plasmodium falciparum gametocytes. mSphere. 2018;3:e00130.

54. Lee HJ, Georgiadou A, Otto TD, Levin M, Coin LJ, Conway DJ, Cunnington AJ. Transcriptomic studies of malaria: a paradigm for investigation of systemic host-pathogen interactions. Microbiol Mol Biol Rev. 2018:82:e00071.

55. Kozarewa I, Ning Z, Quail MA, Sanders MJ, Berriman M, Turner DJ. Amplification-free Illumina sequencing-library preparation facilitates improved mapping and assembly of $(\mathrm{G}+\mathrm{C})$-biased genomes. Nat Methods. 2009;6:291-5.

56. Kim D, Langmead B, Salzberg SL. HISAT: a fast spliced aligner with low memory requirements. Nat Methods. 2015;12:357-60.

57. Li H, Handsaker B, Wysoker A, Fennell T, Ruan J, Homer N, Marth G, Abecasis G, Durbin R, Genome Project Data Processing S. The sequence alignment/ map format and SAMtools. Bioinformatics. 2009;25:2078-9.

58. Lawrence M, Huber W, Pages H, Aboyoun P, Carlson M, Gentleman R, Morgan MT, Carey VJ. Software for computing and annotating genomic ranges. PLoS Comp Biol. 2013;9:e1003118.

59. Love Ml, Huber W, Anders S. Moderated estimation of fold change and dispersion for RNA-seq data with DESeq2. Genome Biol. 2014;15:550

60. Salanti A, Staalsoe T, Lavstsen T, Jensen AT, Sowa MP, Arnot DE, Hviid L, Theander TG. Selective upregulation of a single distinctly structured var gene in chondroitin sulphate A-adhering Plasmodium falciparum involved in pregnancy-associated malaria. Mol Microbiol. 2003;49:179-91.

Ready to submit your research? Choose BMC and benefit from:

- fast, convenient online submission

- thorough peer review by experienced researchers in your field

- rapid publication on acceptance

- support for research data, including large and complex data types

- gold Open Access which fosters wider collaboration and increased citations

- maximum visibility for your research: over $100 \mathrm{M}$ website views per year

At BMC, research is always in progress.

Learn more biomedcentral.com/submissions 